\title{
Magnetocardiogram Interference in Magnetoencephalographic Data: Its Importance in Cases of Interictal Epileptic Data
}

\author{
PD Bamidis ${ }^{1,2}$ \\ ${ }^{1}$ South-East European Research Centre (SEERC), Thessaloniki, Greece, \\ ${ }^{2}$ CITY College, Affiliated Institution of the University of Sheffield, Thessaloniki, Greece
}

\begin{abstract}
In this paper, the importance of heart interference to models of magnetic brain activity as measured by magnetoencephalograms (MEG) of epileptic patients is explored. Cases where this is crucial to examine are used as triggers of this discussion. A distributed source modelling technique of brain activity, namely, Magnetic Field Tomography (MFT) provides the context of the methodology used to certify that results obtained are free from cardiac interferences. Different visualisation strategies are employed to further confirm that. These are time depthplots of source activity and global field power measures in association with ECG traces. Finally, possible ECG artifact rejection methods are discussed.
\end{abstract}

\section{Introduction}

Neuromagnetism studies the magnetic fields emanated from various organs of living organisms (in this case humans). It is obvious that both human brain and heart are sources of such fields. However, the latter are much stronger than the former, meaning that upon modeling magnetic brain activity (Magnetoencephalogram, MEG) one should pay attention to possible interferences from cardiac magnetic activity (Magnetocardiogram, MCG).

Cardiac signals are much stronger than brain signals (at least two to three orders of magnitude). Of course, when the recording probe is placed over the head, the heart is a few tens of centimetres away, and the inverse distance square law (Biot-Savart) partially compensates for the above figure. But, there have been studies of cardiac contamination of real MEG signals recorded in magnetically shielded rooms while subjects were sitting under a neuromagnetometer [1]. When MEG signals were averaged time-locked to the $R$ wave of the electrocardiogram, maximum amplitudes of cardiac artifacts varied but was on average some $130 \mathrm{fT} / \mathrm{cm}$. In all such studies, it was implied that artifacts were generated by cardiac currents, without any significant contribution from blood-flow-related pulsations or body movements. Therefore, such artifacts may have a considerable effect on unaveraged (single epoch) data, i.e., recordings of spontaneous brain activity. Thus, cardiac contamination of MEG signals should be taken into account in the MEG analysis [1].

\subsection{Distributed tomographic modeling of brain neural activity}

Over the past decade distributed source models have been increasingly applied to biomagnetic data. Magnetic Field Tomography (MFT) is one of the first such methods, that makes use of probabilistic estimates of the primary current density $\mathbf{J}_{\mathbf{p}}(\mathbf{r})$, as it is extracted from biomagnetic data [2]. The primary current density is confined to a fixed source space, while the use of lead fields allows a once and for all computation of geometry and conductivity dependent effects. MFT has been used in the analysis of both MEG [3] and MCG data [4].

In other studies, both with MFT and other distributed techniques, it has been shown that emphasis should be placed upon the propagation analysis of brain activity rather than mere static modelling of signal maxima localisation [5]. If the heart activity contributes to (contaminates in this respect) brain activation interplays, it then ought to be identified and removed.

\subsection{Identification and elimination of cardiac contribution in MEG signals: a review}

To reduce physiological artifacts in MEG and EEG recordings, numerous methods have been applied, namely, spatiotemporal matching, principal component analysis, signal-space projection, regression using secondary information, and independent component analysis [6,7].

For instance, in [8], a typical two-step method for identification and elimination of the cardiac contribution in single-trial MEG signals is given. In the first step, the mean interfering signal (MIS) in one period is estimated by QRS-synchronous averaging of the raw MEG data. In the second step, a QRS-synchronous segmentation of the MEG signals is performed and each signal segment is orthogonalized with the MIS. The method is tested in real MEG data, from which cardiac interference is eliminated 
whereas the components of interest (brain activity) remain almost unaffected.

More recently, independent component analysis (ICA) has appeared as a powerful tool in separating signals from their mixtures [9]. In one of the many algorithms proposed, a priori information in a fixed point algorithm was used to find the signal of interest out of a number of sensors, thereby canceling cardiac artifacts from the magnetoencephalogram [10].

\subsection{MFT cases to consider the MCG interference}

Although epileptic MEG signals are quite strong (even in the interictal state), the case for MCG interference should be closely examined when brain activity, as estimated by MFT, appears at the deep (distant to the sensors) levels or the edges of the source space. Figure 1 displays such a case of unilateral measurements when a partial hemispherical source is used [11]. Is such deep activity a mere artifact of cardiac interference or is it something else?
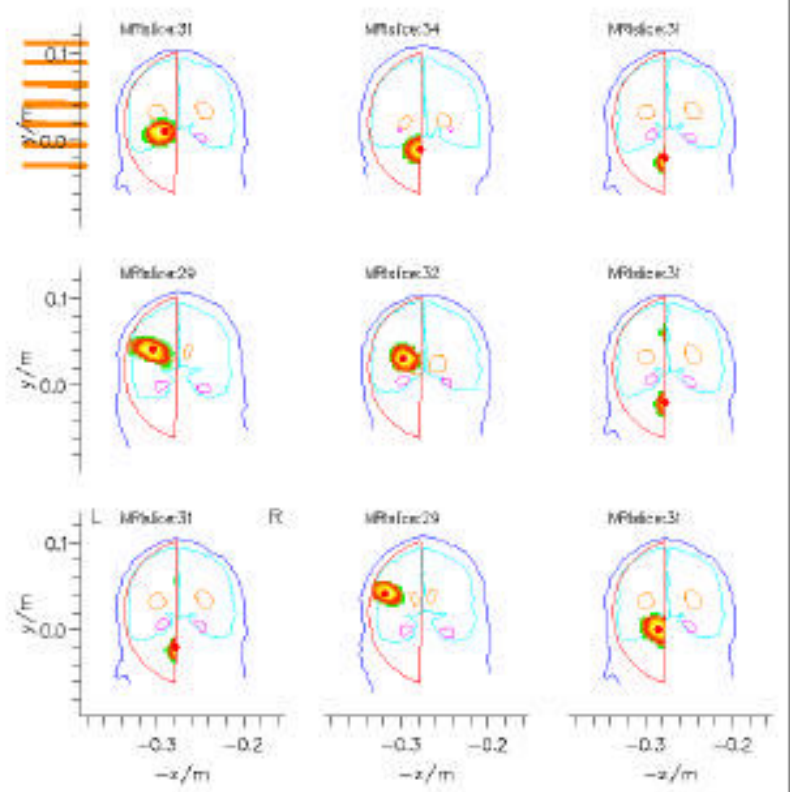

Figure 1. Successive MFT contour plots of the squared current density estimates superimposed on MRI outlines. Sources within the partial hemispherical source space are satisfactorily recovered; when strong generators are present beyond the bottom source space level, solutions appear as "focal" (in this coronal perspective) deep activity.

This paper focuses on the MFT analysis of interictal epileptic MEG data and discusses the importance of MCG interferences in this kind of analysis.

\section{Material and methods}

MEG signals were recorded with a multi-channel system placed over the temporal area of the head of temporal epileptic patients. Simultaneous one-lead recordings of the ECG were also performed (extremity leads).

A number of different approaches are used to resolve such situations. In one of the approaches, a cylindrical source space shifted towards the depth of the brain was utilized to "focus" on the unknown deep activity characteristics $[3,11]$.

In addition to this, MFT estimates are integrated over the area of each source space level and for each time slice separately. The integral values are then represented as time-depthplots by assigning them a pixel and a colour value and arranging the pixels along the various source space levels in a single column. This is repeated for each time slice of considerably long epochs (e.g. 2.5-3 secs) as far as interictal epileptic activity is concerned. The timedepthplots are viewed in association with the ECG signal for a quick qualitative comparison and extraction of the result.

Additional qualitative measures of MCG interference are obtained by studying the correlation of the ECG signal with the global (estimated) density power.

Furthermore, if strong cardiac artifacts are observed different artifact rejection methods may be employed to clean the MEG signals.

\section{Results}

Figure 2 illustrates how the situation in Figure 1 was resolved and deep sources are now attributed to certain anatomical areas rather than source space extremes.
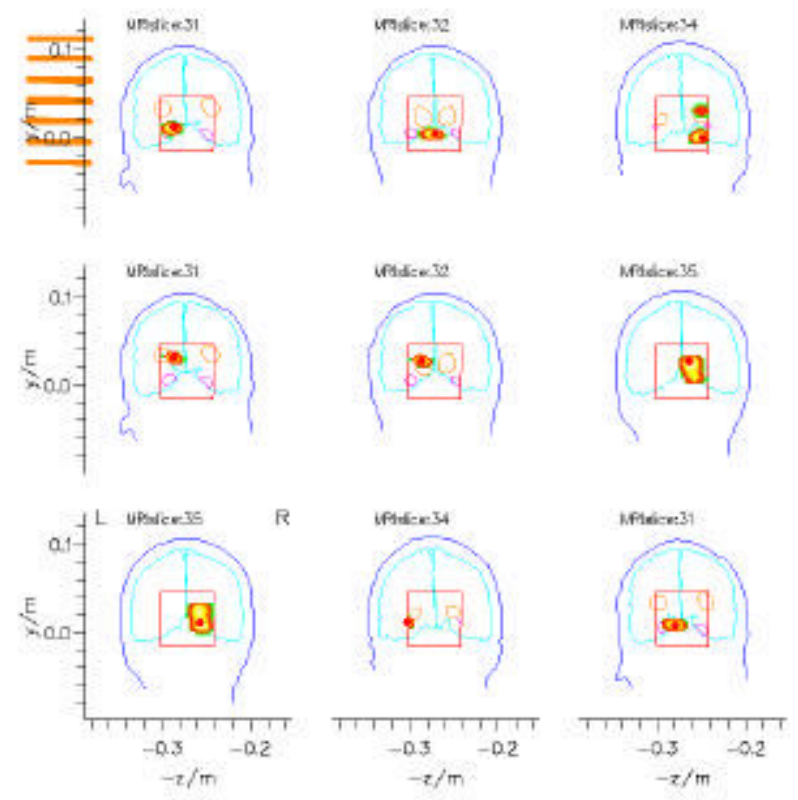

Figure 2. MFT contour plots, when current density estimates are obtained within a centrally - to the head placed small cylinder. One can now see where the "focal deep" activity appearing in Fig. 1 is coming from. 
However, for double-checking purposes, one should investigate the relationship of such activations with the cardiac activity. To do this, the time-depthplots are calculated and displayed in Figure 3 for some signal segment.
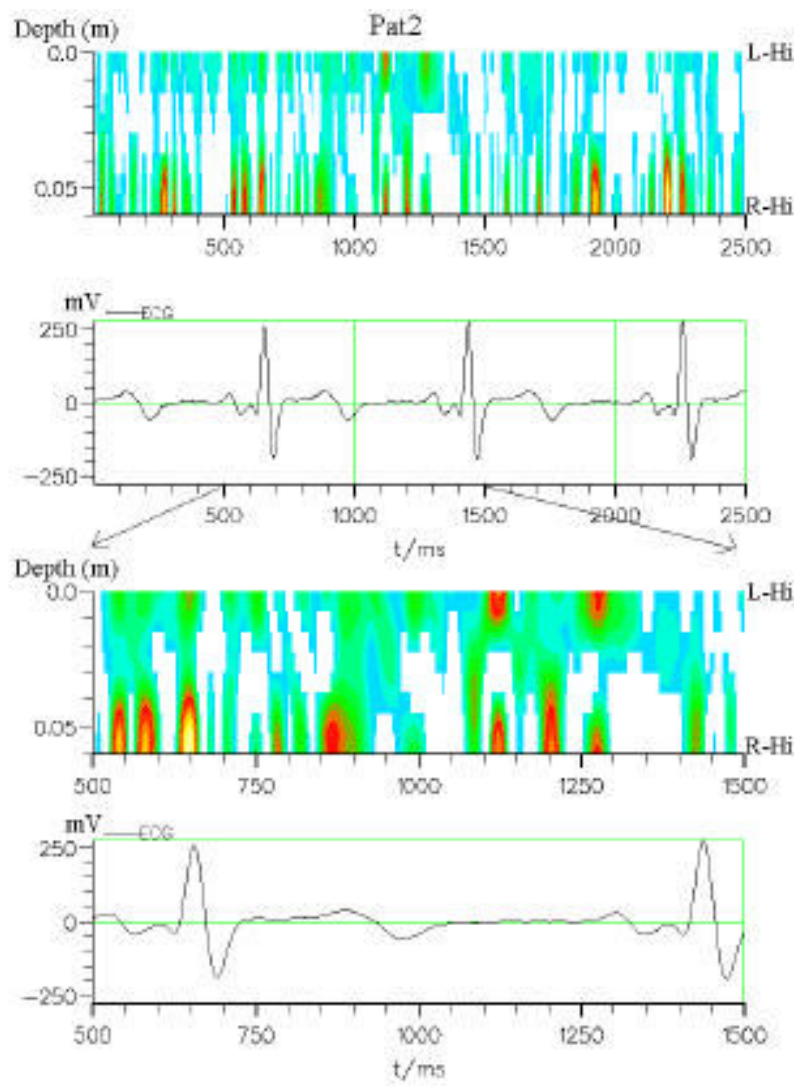

Figure 3. Top half: Time-depthplots for the small cylindrical source space (top) along with the ECG signal (bottom) for one of the patients. Zero depth corresponds to left temporal area, while 5-6 cm depth corresponds to right temporal area. Bottom half: an expanded time segment of the above period. The firing of the right temporal area (deep focal activity in Fig. 1) is clearly independent of the heart cycle.

In addition to the above method, one may calculate the global field power of the MEG signals and compare it with the ECG signal. This is done in Figure 4 for a considerably long epoch segment (7 sec).

Quantitative measures may be obtained by calculating the correlation of the two traces in Figure 3. However, mere observations of the Figure may still provide good qualitative results.

In cases where the above correlation seems to be high (or at least suspicious) MCG artifact rejection methods may be employed to clean the MEG signal. ICA has been used in these data successfully [12], as well as, the spatiotemporal matching described in [7]. Once the heart artifacts are removed, MFT analysis is repeated, in order to realize possible changes. However, changes are not always easily interpretable.
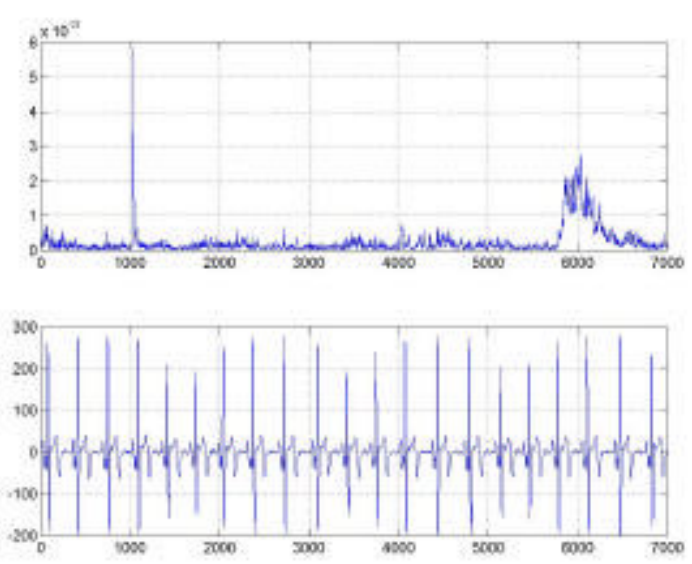

Figure 4. Signal-space glimpses of overall MCG interference on MEG signals. Top half: the global field power of all MEG channels for some $10 \mathrm{sec}$ of the recording time. Bottom half: the ECG record for the same period.

\section{Discussion}

This paper is focused on the importance of MCG interferences in the MFT analysis of interictal epileptic MEG data. It was shown that, in the MEG segments of interest, the MCG artifacts are not significant, and therefore, the obtained results are trustworthy at this level. Although no significant changes were observed in the MFT findings after artifact rejection, however, one needs to be very careful in the analysis and always check for MCG artifacts. Strong candidate cases for the above elaboration were provided in this paper, namely, cases where deep brain activity was observed in the source space. Similar cases may exist when good correlation between the global field power (signal space) and the ECG signal is observed.

Different ways of how to visualize such effects were suggested and different methodologies were proposed for their resolution.

In addition to the above, we have used two different artifact rejection methods (Independent Component Analysis (ICA), and spatiotemporal matching) to clean the MEG signal from heart artifacts, and the MFT analysis was repeated. Results from these attempts are not presented here due to space limitations. It is very important to compare the effectiveness of the different artifact rejection techniques and methodologies, but this goes beyond the purpose of the current paper, however, and will be the objective of another work. Recently, timedelayed decorrelations were combined with ICA, in a straightforward calculation of the correlation between signals having different time shifts with respect to each other [13]. Such techniques may exploit different complex frequency spectra of the sources and may be very useful when assigning the obtained ICA components to cardiac artifacts. 
Finally, ICA based techniques and MCG identification may be used in another context, as well. That is, once the MCG linked ICA components have been identified, their association (correlation) with heart activity may be studied. Then, analysis of their relationship with the rest of the ICA (brain activity related) components [14] will probably provide hints, as to how heart and brain activities are associated in pathological conditions like epilepsy.

\section{References}

[1] Jousmaki V, Hari R. Cardiac artifacts in magnetoencephalogram. J Clin Neurophysiol. 1996; 13(2):172-6.

[2] Ioannides AA. Estimates of $3 \mathrm{~d}$ brain activity ms by ms from biomagnetic signals: method (MFT), results and their significance. In: Eiselt E, et al (Eds), Quantitative and Topological EEG and MEG analysis. Univ.DruckhausMaayer, 1995:59-68.

[3] Bamidis PD, Hellstrand E, Lidholm H, Abraham-Fuchs K, Ioannides AA. MFT in complex partial epilepsy: spatiotemporal estimates of interictal activity. Neuroreport, 1995, 7(1): 17-23.

[4] Ioannides AA, Clewett BS, Weise F, Dammers J, Gross J, Bamidis PD, and Mueller-Gaertner HW. Magnetic Field Tomography analysis of the Magnetocardiogram. In: Book of abstracts, 10th International Conference on Biomagnetism, Santa Fe, USA, 1996:137.

[5] Bamidis PD, and Ioannides AA. Processing of MFT images to reveal the propagation characteristics of epileptic activity. Med. Biol.Eng.Comp. 1999;37:Suppl 2(II): 962-3.

[6] Sander TH, Wubbeler G, Lueschow A, Curio G, Trahms L. Cardiac artifact subspace identification and elimination in cognitive MEG data using time-delayed decorrelation. IEEE Trans Biomed Eng. 2002; 49(4):345-54.

[7] Abraham-Fuchs K, Härer W, Schneider S, and Stefan H. Pattern recognition in biomagnetic signals by spatiotemporal correlation and applications to the localization of propagating neural activity. Med. Biol. Eng. Comput. 1990;28:398-406.
[8] Samonas M, Petrou M, Ioannides AA. Identification and elimination of cardiac contribution in single-trial magnetoencephalographic signals. IEEE Trans Biomed Eng. 1997; 44(5):386-3.

[9] Cardoso J.F. Blind signal separation: statistical principles. Proc.of IEEE 1998; 9:2009-25.

[10] Barros AK, Vigario R, Jousmaki V, Ohnishi N. Extraction of event-related signals from multichannel bioelectrical measurements. IEEE Trans Biomed Eng. 2000; 47(5):5838.

[11] Bamidis PD. Spatio-temporal evolution of Interictal Epileptic Activity: a study with unaveraged multichannel MEG data in association with MRIs. PhD Thesis. Milton Keynes, UK: The Open University, 1996.

[12] Bamidis PD, Zisis A. Studying Epileptic Activity Propagation: From Robust But Time-Consuming To Intelligent Information Processing Methodologies. Control Eng. Applied Informatics Journal, 2002, Vol. 4(1):45-54.

[13] Sander TH, Lueschow A, Curio G. Time-delayed decorrelation for the identification of cardiac artifact components in MEG data. Biomed Tech (Berl). 2002, 47 Suppl 1 Pt 2:573-6.

[14] Bamidis PD, Zisis A, Maglaveras N, Kostopoulos G, and Ioannides AA. Studying interictal epileptic activity propagation with ICA and MFT. In: Biomag 2002: Proceedings of the 13th International Conference on Biomagnetism, August 2002, Jena-Germany, VDE Verlag, Berlin, p.1001-1003.

Address for correspondence.

Dr Panos D. Bamidis

SEERC, South East-European Research Centre 17 Mitropoleos Str., 54624 Thessaloniki, GREECE

E-mail address: pbamidis@ seerc.info 